\title{
Differences in nulliparous caesarean section rates across models of care: a decomposition analysis
}

\author{
Aoife Brick ${ }^{1,2^{*}}$, Richard Layte ${ }^{3,1}$, Anne Nolan ${ }^{1,2}$ and Michael J. Turner ${ }^{4}$
}

\begin{abstract}
Background: To evaluate the extent of the difference in elective (ELCS) and emergency (EMCS) caesarean section (CS) rates between nulliparous women in public maternity hospitals in Ireland by model of care, and to quantify the contribution of maternal, clinical, and hospital characteristics in explaining the difference in the rates.

Methods: Cross-sectional analysis using a combination of two routinely collected administrative databases was performed. A non-linear extension of the Oaxaca-Blinder method is used to decompose the difference between public and private ELCS and EMCS rates into the proportion explained by the differences in observable maternal, clinical, and hospital characteristics and the proportion that remains unexplained.

Results: Of the 29,870 babies delivered to nulliparous women, 7,792 were delivered via CS (26.1\%), $79.6 \%$ of which were coded as EMCS. Higher prevalence of ELCS was associated with breech presentation, other malpresentation, and the mother being over 40 years old. Higher prevalence of EMCS was associated with placenta praevia or placental abruption, diabetes (pre-existing and gestational), and being over 40 years old. The private model of care is associated with ELCS and EMCS rates 6 percentage points higher compared than the public model of care but this differential is insignificant in the fully adjusted models for EMCS. Just over half (53\%) of the 6 percentage point difference in ELCS rates between the two models of care can be accounted for by maternal, clinical and hospital characteristics. Almost $80 \%$ of the difference for EMCS can be accounted for.

Conclusions: The majority of the difference in EMCS rates across models of care can be explained by differing characteristics between the two groups of women. The main contributor to the difference was advancing maternal age. The unexplained component of the difference for ELCS is larger; an excess private effect remains after accounting for maternal, clinical, and hospital characteristics. This requires further investigation and may be mitigated in future with the introduction of clinical guidelines related to CS.
\end{abstract}

Keywords: Elective caesarean section, Emergency caesarean section, Private practice, Non-linear Oaxaca-Blinder decomposition method

\section{Background}

In recent decades a feature of modern obstetric practice in developed countries has been the relentless rise in caesarean section (CS) rates. The reasons for the increase in CS rates across countries are multiple and complex but have been attributed to the increasing prevalence of older mothers, rising rates of maternal

\footnotetext{
* Correspondence: aoife.brick@esri.ie

${ }^{1}$ Economic and Social Research Institute, Whitaker Square, Sir John Rogerson`s Quay, Dublin 2, Ireland

${ }^{2}$ Trinity College, Dublin, Ireland

Full list of author information is available at the end of the article
}

obesity, medical comorbidities, and changing medical practice including the increasing relative safety of CS itself [1-4].

While CS rates are rising globally, there is substantial evidence that this increase is more prevalent amongst women with privately funded deliveries [5-11]. This raises questions of whether CS is always medically indicated in privately funded patients $[7,8]$. There is an important issue of confounding in this scenario however. Women with a higher risk of complicated pregnancies and deliveries due to older maternal age and other 
relevant maternal and clinical risk factors are more likely to take out private health insurance (PHI) due to perceived quality of care [12-17]. The positive association between private care and CS may thus reflect the selection of women with clinically complex deliveries into private care rather than measuring the effect of private status on the process leading to CS.

As well as women selecting private status there may also be clinician/ organisational level factors at play. For example, with high numbers of deliveries to contend with the ability to be able to schedule deliveries for private patients may become increasingly attractive [18]. In addition, in an increasingly litigious society the level of risk a clinician deems acceptable may be lower, particularly so for private patients [19].

Models of obstetric care in Ireland are unusual by international standards in that private patients are looked after by the same midwifery staff in the wards, including the labour ward, as public patients. Furthermore, the same clinical guidelines are applied across models of care. Nonetheless, variation in obstetric interventions across models of care has been found. The rate of increase in CS for women funded publicly was $7.2 \%$ (22.2 \% to $23.8 \%$ ) between 2005 and 2010 in Ireland compared to $14.9 \%$ (30.2 \% to $34.7 \%$ ) for those funded privately [19]. Irish research suggests that the higher rate of CS for privately funded births cannot be explained by differences in medical or obstetric risk factors between women but no study to date has employed national, individual data to test this hypothesis [20, 21].

Research internationally has suggested that financial incentives may increase the probability of CS $[9,22,23]$ but the payment method for private care in Irish hospitals and hospital financing system means that the financial benefit from higher CS rates does not accrue to the hospital. In fact, the annual financial allocation to hospitals is reduced as income from private practice increases.

Previous literature has described the increasing prevalence of CS and the role of the private model of care in this. This paper contributes to the literature by quantifying the extent to which the differences in CS rates across models of care is due to differences in maternal, clinical, and hospital characteristics. To reduce the heterogeneity of the sample and the complexity of the processes that we study, this paper focuses solely on nulliparous women.

Section 2 describes the data, Section 3 outlines the methods, Section 4 presents empirical results, and Section 5 discusses the findings and concludes.

\section{Methods}

Data

The data for these analyses were obtained from two national databases in the Republic of Ireland, the National
Perinatal Reporting System (NPRS) and the Hospital InPatient Enquiry (HIPE) scheme. The main source of data on perinatal events in Ireland is the NPRS which contains information on all deliveries ( $\geq 500 \mathrm{~g})$ in the Republic of Ireland. Unfortunately, clinical data on deliveries are not available in the NPRS which means that it is not possible to identify the clinical indicators for CS using these data, including whether the CS was carried out on an elective or emergency basis.

However, data on these variables are available from the HIPE system. The HIPE system records data on all discharges from, and deaths in, acute publicly funded hospitals in the Republic of Ireland. These data contain information on administrative data such as admission and discharge dates and model of care (public/private) as well as maternal and child demographic data and clinical information. The clinical data on discharges in 2009 were recorded in HIPE using the 6th Edition of The International Statistical Classification of Diseases and Related Health Problems, Tenth Revision, Australian Modification (ICD-10-AM), the Australian Classification of Health Interventions (ACHI) and the Australian Coding Standards (ACS). Deliveries in HIPE are identified by the presence of a diagnosis of outcome of delivery (ICD-10-AM - Z37). In total, up to 20 diagnosis codes (one principal and up to 19 additional) and, where applicable, 20 procedure codes (one principal and up to 19 additional) could be recorded for these discharges.

The sample employed for these analyses consists of all nulliparous singleton births to women discharged from the 19 publicly funded hospital maternity units between 01 January 2009 and 31 December 2009 and for whom an NPRS and HIPE record were available; a total of 29,870 births. This represented $96.7 \%$ of the total number of nulliparous singleton births in publicly funded hospital maternity units in 2009. For these analyses, only births (live and stillborn) that took place within a hospital were included, that is, cases where 'place of birth' is recorded as 'domiciliary,' 'born before arrival' and 'unknown' are excluded.

\section{Dependent variables}

The dependent variables are binary indicators of whether the mother had a CS (elective or emergency) or not. In ACS (1541) an ELCS (ACHI 16520-00, 16520-02) is defined as a CS carried out as a planned procedure before the onset of labour or following the onset of labour, when the decision was made before labour. An EMCS (ACHI 16520-01, 16520-03) is defined as a CS required because of an emergency situation. It is best described as 'when the CS is performed having not been considered necessary previously' [24]. According to the European Perinatal 
Health Report in 2010 these definitions are commonly used in other countries [25].

\section{Independent variables}

Independent variables include those familiar from previous research on CS determinants [3]. Data on the woman's marital status, socio-economic group, country of birth, birthweight, gestational age, and obstetric history were sourced from NPRS. Data on the woman's age, public/private status, method of delivery, and clinical risk factors for CS were sourced from HIPE. Socioeconomic group is derived from information on maternal occupation, and coded, with minor modifications, using the schema employed by the Central Statistics Office. The clinical risk factors for CS included in the analysis are those commonly used throughout the literature (see Additional file 1 for codes). Fetal distress and dystocia have been excluded as indicators for EMCS. A variable representing whether the woman has previously experienced a miscarriage is included to measure the possible selection of women into private care as well as the direct correlation between past miscarriage and CS currently.

The main independent variable of interest is model of care which refers in these data to whether the woman was a public or private patient of their chosen consultant and not to the type of bed occupied. All women in Ireland are entitled to free maternity services but a proportion choose to finance their care privately through PHI and/or an 'out-of-pocket' payment. A public patient typically receives shared care from their family doctor and their chosen hospital. They may or may not see the same obstetrician on each of their antenatal visits to the hospital and after delivery is moved to a shared room (usually four or six bedded).

Being a private patient in a publicly funded hospital means that you are the private patient of your chosen consultant who will supervise your antenatal care. The consultant or a nominated consultant colleague will also attend for delivery. After delivery, patients are transferred to a private room if one is available. Some hospitals also offer a semi-private option, which usually means patients antenatal care alternates between their chosen consultant and their GP. Their delivery will be attended by the obstetrician on duty and they are transferred to a semi-private room following delivery if one is available. In HIPE, unfortunately both private and semi-private patients are classified as private and in the 2009 data it is not possible to distinguish between the two. This could mean that the effect of private care is reduced if the processes associated with private care among women do not apply to semi-private care.

\section{Statistical analysis}

\section{Determinants of CS}

We first analyse the determinants of ELCS and EMCS by estimating the following non-linear model:

$$
Y_{i}=F\left(X_{i} \beta\right)
$$

where $y_{i}$ is the dummy variable which indicates whether the woman had a CS and $X_{i}$ is a vector of maternal and clinical characteristics.

\section{Decomposition of the difference in cs rates across models of care}

To examine the extent to which the differences in the rates of ELCS and EMCS across models of care is due to differences in their observed characteristics, a non-linear approximation of the Blinder-Oaxaca decomposition technique is used [26-28]. The technique is used to study group differences in an outcome variable and has been employed in analyses of racial/ethnic differences in birthweight; child mortality; child health insurance cover; life expectancy; and breastfeeding [29-34].

The average difference in the CS rates between public and private women may be expressed as:

$$
\begin{aligned}
& \bar{Y}^{p u b}-\bar{Y}^{p r i}=\left[\sum_{i=1}^{N^{p u b}} \frac{F\left(X_{i}^{p u b} \hat{\beta}^{p u b}\right)}{N^{p u b}}-\sum_{i=1}^{N^{p r i}} \frac{F\left(X_{i}^{p r i} \hat{\beta}^{p u b}\right)}{N^{p r i}}\right] \\
& +\left[\sum_{i=1}^{N^{p r i}} \frac{F\left(X_{i}^{p r i} \hat{\beta}^{p u b}\right)}{N^{p r i}}-\sum_{i=1}^{N^{p r i}} \frac{F\left(X_{i}^{p r i} \hat{\beta}^{p r i}\right)}{N^{p r i}}\right]
\end{aligned}
$$

where $\bar{Y}^{J}$ is the average probability of CS for group $J$ $(J=p u b, p r i) X_{i}^{J}$ is the vector of independent variables of observation $i$ in group $J, \hat{\beta}^{J}$ is the vector of coefficient estimates and $N^{J}$ is the number of observations in group $J$. In this case, group pub is the sample of public mothers, group pri is the sample of private mothers, and the reference is group pub. We also undertake the decomposition using the estimated coefficients of group pri and the pooled coefficients as the reference $[29,31,35,36]$.

In this application, the first term on the right hand side of (2) measures the amount of the gap in the CS rate that is due to differences in the characteristics of the two groups. The second term captures the degree to which public and private mothers with similar observable characteristics have different CS rates. This may be interpreted as reflecting, varying obstetric practice, group-specific attitudes, or other omitted variables. The first part may be further decomposed into the relative contributions of each of the observed independent variables. We use the 'Fairlie' decomposition command in 
STATA 13.1 [37] with randomly ordered variables and 1,000 replications [38].

\section{Results}

Table 1 shows prevalence and unadjusted CS rates by model of care by maternal, clinical, and hospital characteristics. There were higher proportions of private patients in the older age groups. Over $80 \%$ of private patients were in the professional/managerial or clerical social classes $(p<0.01)$. Only $45 \%$ of public women were married compared to $83.5 \%$ of private women $(p<0.01)$. Over $92 \%$ of private women were born in Ireland compared to $64 \%$ of public women. Of the public women born outside Ireland, $20.9 \%$ were from the EU-27 countries $(p<0.01)$.

Almost $18 \%$ of private women had a previous miscarriage compared to $13.3 \%$ of public women $(p<0.01)$. Women in the public group had smaller babies than those in the private group $(p<0.01)$. Many of the clinical characteristics varied significantly by model of care including breech presentation, diabetes mellitus (preexisting and gestational), other malpresentation, placenta praevia or placental abruption and induction of labour. A majority of private women $(60.9 \%)$ gave birth in an academic teaching hospital compared to public women $(47.5 \%)(p<0.01)$.

A key objective of this research is to examine the extent to which the difference in CS rates across models of care can be accounted for by the differences in maternal, clinical, and hospital characteristics. That is, do private women have higher CS rates primarily because they have socio-demographic and clinical characteristics that are associated with higher $\mathrm{CS}$ rates?

Figure 1 shows that there is a 6 percentage point difference between both the ELCS and EMCS rates for public and private women. The difference between rates varied across model of care with the presence of various characteristics. For ELCS the private rate is higher than the public rate for almost all characteristics. The differences are particularly large for the clinical risk factors. For EMCS there is more variation with the public rate being higher than the private rate for some indications. For example, the public ELCS rate for women with other malpresentation is $35.4 \%$ compared to $70.9 \%$ for private women. In contrast, for the same indication, the EMCS rate is $43.5 \%$ for public women and $23.4 \%$ for private women.

\section{Determinants of CS}

The results from the probit models for ELCS and EMCS follow a similar pattern to the descriptive statistics discussed earlier. Table 2 shows that in the fully adjusted models, women who are privately funded have a significantly higher probability of ELCS compared to publicly funded women, but also that private status has no significant effect on EMCS risk. The probability of both EMCS and ELCS increases significantly with maternal age and where mother is defined as holding a clerical or unskilled occupation or is a full-time carer relative to women with a professional occupation. In addition, being unemployed increases the probability of ELCS but has no effect on EMCS.

Not being married is associated with an increased probability of EMCS compared to married woman. Women born in Africa have a significantly higher risk of both EMCS and ELCS whilst women from Asia are at a higher risk of EMCS but a significantly lower risk of ELCS relative to women from Ireland. Being from EU27 Accession States significantly lowers the probability of EMCS. Women born in the UK have a significantly higher probability of having an ELCS compared to Irish-born women.

Having had a previous miscarriage had a significant positive impact on the probability of EMCS. Birthweight and all included clinical risk factors have significantly positive effects on the probability of both ELCS and EMCS with the exception of hypertensive disorders on ELCS which has no effect. Delivering in an academic teaching hospital significantly reduces the probability of having an EMCS but has no significant effect on ELCS.

\section{Decomposition of the difference in CS rates across models of care}

To investigate if the socio-demographic and clinical risk profile of private women accounts for their higher risk of CS, a decomposition of the difference in ELCS and EMCS rates between public and private women was undertaken, the results of which are presented in Table 3. The raw difference in CS rates is 6.1 percentage points for ELCS and 6.0 percentage points for EMCS, with private women having the higher rates. The results show that the distribution of age, breech presentation and malpresentation between private and public women accounts for $53.3 \%$ of the difference in ELCS rate and $79.7 \%$ of the difference in the EMCS rate. Examining the results using different reference coefficients reveals that while the size of the explained contribution differs (ELCS private $71.1 \%$, pooled $54.9 \%$; EMCS private $87.9 \%$, pooled $78.2 \%)$, the differences are not large, and the main conclusions still hold [28, 31, 35].

Given the large proportion of the EMCS difference accounted for by age, sensitivity analysis was carried out to test the relationship of the effect of age to the clinical risk factors in our models. Two additional models were run, one excluding clinical characteristics and the second excluding age (available from the authors on request). Excluding clinical characteristics 
Table 1 Prevalence and Unadjusted Caesarean Section Rates (Elective and Emergency) for Nulliparous Singleton Deliveries by Maternal and Clinical Characteristics, 2009

\begin{tabular}{|c|c|c|c|c|c|c|c|}
\hline & \multirow{2}{*}{\multicolumn{3}{|c|}{ Prevalence }} & \multicolumn{4}{|c|}{ CS Rate ${ }^{a}$} \\
\hline & & & & \multicolumn{2}{|c|}{ Elective } & \multicolumn{2}{|c|}{ Emergency } \\
\hline & Public & Private & $p$-value & Public & Private & Public & Private \\
\hline$N$ & 22,059 & 7,811 & & 913 & 664 & 4,325 & 1,890 \\
\hline \multirow[t]{2}{*}{ CS Rate } & 23.7 & 32.7 & & 4.1 & 8.5 & 19.6 & 24 \\
\hline & $\%$ & $\%$ & & $\%$ & $\%$ & $\%$ & \\
\hline
\end{tabular}

Maternal Characteristics

Age (years)

$<20$

20-24

25-29

30-34

35-39

$\geq 40$

Social Class

Professional/managerial

Clerical

Skilled/semi-skilled

Unskilled

Unemployed

Home duties

Other $^{\mathrm{b}}$

Marital Status

Married

Not married

Country of Birth

Ireland

UK

EU-15

EU-27 ${ }^{d}$

Africa

Asia

Other $^{\mathrm{e}}$

Clinical Characteristics

Obstetric History

Previous miscarriage

Gestational Age (weeks)

$<33$

33-37

$38+$

Birthweight (g)

500-2499

2500-2999

3000-3499
8.9

25.0

34.2

22.9

7.9

1.2

21.2

26.0

7.3

18.2

4.6

15.1

7.5

44.9

55.1

64.2

2.4

1.8

20.9

2.4

5.9

2.4

13.3

1.4

7.8

90.8

5.0

13.9

36.5

$$
\begin{array}{r}
0.5 \\
1.8 \\
17.8 \\
52.5 \\
23.3 \\
4.1
\end{array}
$$

$\begin{aligned} 56.5 & 0.000^{* * *} \\ 27.9 & \\ 3.5 & \\ 7.3 & \\ 0.2 & \\ 1.9 & \\ 2.8 & \end{aligned}$

83.5

16.5

$0.000^{* * *}$

$$
92.1
$$$$
1.6
$$

1.1

1.7

17.9

1.2

7.9

90.9

$$
\begin{array}{r}
4.1 \\
11.2
\end{array}
$$$$
34.5
$$

$0.000^{* * *}$

0.460

$0.000^{* * *}$

$$
0.000^{* * *}
$$

2.1

2.1

2.6

3.8

5.1

7.6

19.6

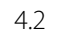

4.9

4.9

4.9

4.1

3.6

3.4

2.5

4.9

3.5

4.3

5.4

5.7

3.8

3.2

3.2

4.1

5.8

\section{6}

5.2

4.0

6.4

4.5

4.6

0.0

4.4

5.5

7.5

10.9

23.2

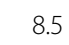

9.0

9.0

9.9

7.9

$$
5.9
$$

6.9

5.1

8.8

7.2

21.2

18.4

12.0

16.2

18.5

23.5

30.7

31.7

21.7

20.3

19.1

19.8

16.1

19.0

14.9

$\%$

$\%$$$
\text { 更 }
$$

. 
Table 1 Prevalence and Unadjusted Caesarean Section Rates (Elective and Emergency) for Nulliparous Singleton Deliveries by Maternal and Clinical Characteristics, 2009 (Continued)

\begin{tabular}{|c|c|c|c|c|c|c|c|}
\hline 3500-3999 & 32.4 & 35.3 & & 3.2 & 7.8 & 18.9 & 22.4 \\
\hline $4000-4499$ & 10.5 & 12.5 & & 3.1 & 7.2 & 29.1 & 31.4 \\
\hline $4500+$ & 1.7 & 2.4 & & 6.9 & 13.6 & 40.8 & 46.7 \\
\hline \multicolumn{8}{|l|}{ Clinical Risk Factors $^{f}$} \\
\hline Breech presentation & 4.1 & 4.9 & $0.005^{* * *}$ & 66.0 & 72.6 & 29.2 & 25.3 \\
\hline Diabetes mellitus (pre-existing) & 0.3 & 0.2 & $0.025^{* *}$ & 10.7 & 35.7 & 50.7 & 50.0 \\
\hline Gestational diabetes mellitus & 1.7 & 1.0 & $0.000^{* * *}$ & 6.8 & 23.8 & 33.7 & 26.3 \\
\hline Eclampsia including pre-eclampsia & 3.2 & 3.5 & 0.217 & 5.2 & 9.3 & 43.5 & 52.6 \\
\hline Other malpresentation & 0.7 & 2.0 & $0.000^{* * *}$ & 35.4 & 70.9 & 43.5 & 23.4 \\
\hline Placenta praevia or placental abruption & 0.6 & 0.9 & $0.051^{*}$ & 20.4 & 47.8 & 59.2 & 47.8 \\
\hline Hypertensive disorders & 4.7 & 5.0 & 0.450 & 3.3 & 10.1 & 31.3 & 32.6 \\
\hline Poor fetal growth & 2.5 & 2.3 & 0.353 & 10.4 & 15.6 & 32.0 & 33.3 \\
\hline Induction of labour & 28.9 & 34.4 & $0.000^{* * *}$ & & & 27.3 & 32.9 \\
\hline \multicolumn{8}{|l|}{ Hospital Characteristics } \\
\hline Academic teaching hospital & 47.5 & 60.9 & $0.000^{* * *}$ & 4.0 & 8.5 & 18.8 & 21.6 \\
\hline
\end{tabular}

Percentages columns subject to rounding. Missing values are excluded from the calculation of percentages. ${ }^{* * *} p<0.01,{ }^{* *} p<0.05,{ }^{*} p<0.1$

${ }^{\mathrm{a}} \mathrm{CS}$ rates are calculated as a proportion of total deliveries

b Includes farmers and farm managers, other agricultural occupations and fisheries workers, and not classifiable

Includes never married, divorced, separated, and widowed

${ }^{\mathrm{d}}$ Accession States

eIncludes the Rest of Europe, the Americas, Australia, New Zealand (incl. Oceania), multi-nationality, non-Irish, and no nationality

${ }^{f}$ Clinical risk factors imply that the relevant diagnosis code(s) appeared as one of the up to 20 diagnosis codes (one principal and up to 19 additional) recorded in HIPE, a woman may have more than one clinical risk factor. The presence of a particular risk factor does not imply that this was the 'cause' of the CS

Source: HIPE/NPRS 2009 data

increased the proportion explained by the model to $87.3 \%$ with the proportion explained by age also increasing. The proportion explained increases because the prevalence of each risk factor is higher for women in the public model of care. By excluding age from the model the proportion of the difference explained by the model almost halves to $44.7 \%$. The proportion explained by the remaining characteristics does not change greatly. These analyses suggest that the effect of age is independent of the effect of the clinical risk factors and that the latter does not explain the age effect.

\section{Discussion}

In Ireland and internationally studies have found that women treated privately have a higher risk of both

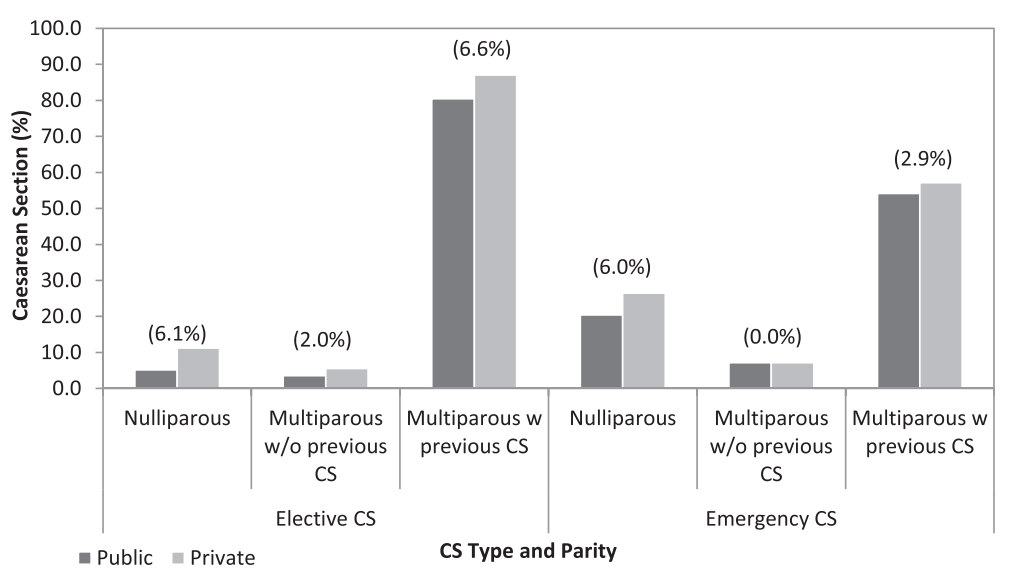

Fig. 1 Elective and Emergency Caesarean Section by Model of Care and Parity, 2009. Notes: Percentage point differences between the public and private rates in parentheses. The ELCS rate is calculated here excluding EMCS and vice versa. Source: HIPE/NPRS 2009 data 
Table 2 Determinants of Caesarean Section (Elective and Emergency) at Hospital Discharge, 2009

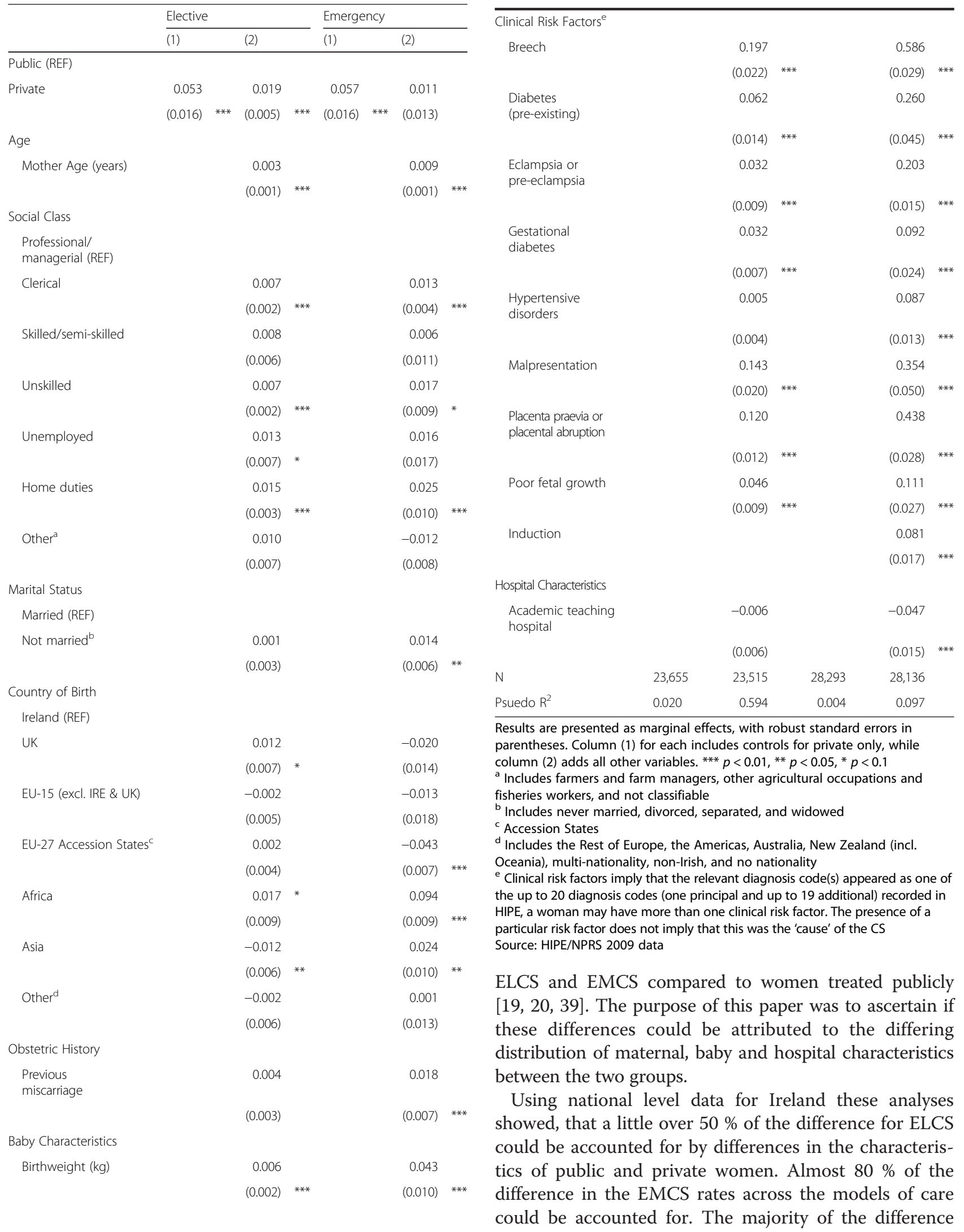

Table 2 Determinants of Caesarean Section (Elective and Emergency) at Hospital Discharge, 2009 (Continued)

Results are presented as marginal effects, with robust standard errors in

a Includes farmers and farm managers, other agricultural occupations and

fisheries workers, and not classifiable

b Includes never married, divorced, separated, and widowed

Accession States

Oceania), multi-nationality, non-Irish, and no nationality

the up to 20 diagnosis codes (one principal and up to 19 additional) recorded in

HIPE a woman may have more than one clinical risk factor. The presence of

particular risk factor does not imply that this was the 'cause' of the CS

ELCS and EMCS compared to women treated publicly $[19,20,39]$. The purpose of this paper was to ascertain if these differences could be attributed to the differing distribution of maternal, baby and hospital characteristics between the two groups.

Using national level data for Ireland these analyses showed, that a little over $50 \%$ of the difference for ELCS could be accounted for by differences in the characterisdifference in the EMCS rates across the models of care The majority of th 
Table 3 Decomposition of the Differential in Elective and Emergency Caesarean Section Rates between Public and Private Mothers

\begin{tabular}{|c|c|c|c|c|c|c|c|c|}
\hline & \multicolumn{4}{|l|}{ Elective } & \multicolumn{4}{|c|}{ Emergency $^{a}$} \\
\hline & $\%$ pt diff & $\%$ of $\bar{Y}^{\text {pub }}-\bar{Y}^{\text {pri }}$ & \multicolumn{2}{|l|}{ z-stat } & $\%$ pt diff & $\%$ of $\bar{Y}^{p u b}-\bar{Y}^{p r i}$ & \multicolumn{2}{|l|}{ z-stat } \\
\hline$\overline{\bar{Y}}^{p u b}-\bar{Y}^{p r i}$ & -0.061 & & & & -0.060 & & & \\
\hline Explained $^{b}$ & -0.032 & 53.3 & & & -0.047 & 79.7 & & \\
\hline$U_{n e x p l a i n e d}^{c}$ & -0.028 & 46.7 & & & -0.012 & 20.3 & & \\
\hline Mother Age & -0.012 & 18.9 & -7.8 & $* * *$ & -0.048 & 80.6 & -14.9 & $* * *$ \\
\hline \multicolumn{9}{|l|}{ Social Class } \\
\hline \multicolumn{9}{|l|}{ Professional/managerial (REF) } \\
\hline Clerical & 0.000 & 0.3 & -1.1 & & 0.000 & 0.4 & -1.3 & \\
\hline Skilled/semi-skilled & 0.000 & -0.7 & 1.5 & & 0.000 & -0.7 & 0.9 & \\
\hline Unskilled & 0.001 & -1.4 & 1.7 & * & 0.002 & -4.0 & 2.3 & $* *$ \\
\hline Unemployed & 0.000 & -0.8 & 1.4 & & 0.001 & -1.0 & 0.9 & \\
\hline Home duties & 0.002 & -3.4 & 2.7 & $* * *$ & 0.004 & -6.5 & 2.9 & $* * *$ \\
\hline Other $^{d}$ & 0.000 & -0.8 & 1.6 & * & 0.000 & 0.5 & -0.6 & \\
\hline \multicolumn{9}{|l|}{ Marital Status } \\
\hline \multicolumn{9}{|l|}{ Married (REF) } \\
\hline Not married ${ }^{e}$ & -0.001 & 1.0 & -0.6 & & 0.005 & -7.7 & 1.9 & * \\
\hline \multicolumn{9}{|l|}{ Country of Birth } \\
\hline \multicolumn{9}{|l|}{ Ireland (REF) } \\
\hline UK & 0.000 & -0.1 & 0.6 & & 0.000 & 0.3 & -1.2 & \\
\hline EU-15 (excl. IRE \& UK) & 0.000 & 0.0 & 0.0 & & 0.000 & -0.1 & 0.5 & \\
\hline EU-27 Accession States ${ }^{f}$ & 0.000 & 0.7 & -0.7 & & -0.008 & 13.7 & -6.3 & $* * *$ \\
\hline Africa & 0.000 & -0.8 & 1.5 & & 0.002 & -4.1 & 4.9 & $* * *$ \\
\hline Asia & 0.000 & 0.6 & -2.0 & $* *$ & 0.001 & -2.2 & 1.9 & * \\
\hline Other $^{9}$ & 0.000 & 0.2 & -1.5 & & 0.000 & 0.4 & -1.6 & \\
\hline \multicolumn{9}{|l|}{ Obstetric History } \\
\hline Previous miscarriage & 0.000 & 0.4 & -1.7 & * & -0.001 & 1.3 & -2.0 & $* *$ \\
\hline \multicolumn{9}{|l|}{ Baby Characteristics } \\
\hline Birthweight & -0.001 & 1.5 & -2.9 & $* * *$ & -0.004 & 6.4 & -8.7 & $* * *$ \\
\hline \multicolumn{9}{|l|}{ Clinical Risk Factors ${ }^{\mathrm{h}}$} \\
\hline Breech & -0.009 & 14.8 & -36.6 & $* * *$ & 0.000 & -0.2 & 1.6 & \\
\hline Diabetes (pre-existing) & 0.000 & -0.1 & 1.0 & & 0.001 & -0.9 & 5.5 & $* * *$ \\
\hline Eclampsia or pre-eclampsia & 0.000 & -0.6 & 1.9 & * & -0.001 & 1.6 & -8.8 & $* * *$ \\
\hline Gestational diabetes & 0.001 & -0.8 & 3.0 & $* * *$ & 0.001 & -1.8 & 4.9 & $* * *$ \\
\hline Hypertensive disorders & 0.000 & 0.0 & -0.1 & & 0.000 & 0.5 & -3.7 & $* * *$ \\
\hline Malpresentation & -0.013 & 21.5 & -19.1 & $* * *$ & -0.001 & 1.0 & -5.0 & $* * *$ \\
\hline Placenta praevia or placental abruption & -0.002 & 3.7 & -9.0 & $* * *$ & 0.000 & -0.2 & 1.2 & \\
\hline
\end{tabular}


Table 3 Decomposition of the Differential in Elective and Emergency Caesarean Section Rates between Public and Private Mothers (Continued)

\begin{tabular}{lcccccc}
\hline $\begin{array}{l}\text { Poor fetal growth } \\
\text { Induction }\end{array}$ & 0.000 & 0.0 & 0.1 & 0.000 & -0.2 & 1.5 \\
Hospital Characteristics & & & -0.007 & 12.0 & -12.0 & $* * *$ \\
Academic teaching hospital & 0.000 & -0.8 & 1.1 & 0.006 & -9.6 & 6.8 \\
\hline
\end{tabular}

Results are presented as marginal effects, with robust standard errors in parentheses

Using the public (pub) coefficients as the reference. Using the private (pri) coefficients as the reference, the explained components were $84.4 \%$ for elective and

$82.2 \%$ for emergency, while using the pooled coefficients results in an explained component of $58.6 \%$ for elective and $78.3 \%$ for emergency (full results available on request from the authors)

*** $p<0.01, * * 0<0.05, * p<0.1$

a Robustness checks were carried out on the EMCS model by excluding clinical characteristics and the proportion explained by the model increased (87.3\% explained). In particular the proportion explained by age and EU-27. The EMCS model was also run excluding age and the proportion explained by the model halves ( $44.7 \%$ explained). This highlights that age explains a large proportion of the difference between public and private EMCS rates and when removed from the model it becomes much less powerful. This is not to say that age is not picking up some omitted variables such as BMI. Results available on request

${ }^{\mathrm{b}}$ The differential that is estimated based on differences in observed characteristics (i.e., the first term in Equation (2))

c The differential that is estimated based on differences in omitted variables e.g. BMI, smoking, etc. (i.e., the second term in Equation (2))

${ }^{d}$ Includes farmers and farm managers, other agricultural occupations and fisheries workers, and not classifiable

e Includes never married, divorced, separated, and widowed

${ }^{f}$ Accession States

${ }^{9}$ Includes the Rest of Europe, the Americas, Australia, New Zealand (incl. Oceania), multi-nationality, non-Irish, and no nationality

${ }^{\mathrm{h}}$ Clinical risk factors imply that the relevant diagnosis code(s) appeared as one of the up to 20 diagnosis codes (one principal and up to 19 additional) recorded in HIPE, a woman may have more than one clinical risk factor. The presence of a particular risk factor does not imply that this was the 'cause' of the CS

Source: HIPE/NPRS 2009 data

for EMCS is accounted for by differences in average maternal age between women experiencing public and private models of care.

The difference in the prevalence of ELCS between women experiencing public and private models of care thus largely reflects the decisions of some women to choose the private model of care and the subsequent impact age has on clinical choices by the obstetrician and the care pathway that the woman follows. Indeed, women may choose private care precisely because they perceive that they will be more able to make choices around their pregnancy and delivery in the private model.

Women using the private model of care tend to be older on average because of their higher levels of education and occupational attainment (the latter shown in Table 1). Over the past decades average age at first birth in Ireland has increased, from 26.3 years in 1990 to 30.2 years in 2013 , largely as a result of the higher proportion of women participating in third level education (Fig. 2). Women are staying in education for longer and are older when they are established in the labour market and in a position where they then choose to have children. Older age at first birth has led to a fall in maternal parity (Fig. 2). Increased complications associated with older maternal age are counter-balanced to some extent by the advantaged socio-economic profile of this group but nonetheless, older age at birth contributes in large part to the higher prevalence of both ELCS and EMCS among women using the private model.

There may also be other factors which could explain the difference in prevalence of CS between women using public and private models for which we do not have measures in our data set. These include maternal pre-pregnancy weight, pregnancy weight gain, fertility treatment, and staff present at delivery [12, 40-43]. However, their inclusion in the models would only increase the proportion explained if these factors were more prevalent in private patients and associated with a higher probability of CS. High pre-pregnancy weight is not actually more prevalent in private patients and although fertility treatment is more prevalent in private patients the proportions are low [20]. A second possible explanation is that there may be behavioural differences on the part of private women and/or their obstetrician that could account for these differences, although this does assume that these behaviours are not correlated with any of the characteristics analysed here.

A limitation of the data is the current lack of availability of data post-2009 that contains all of the variables required to repeat the analysis for a more recent time period. There has been a number of developments post2009 that may have had an impact on the difference in CS rates across models of care.

Firstly, between 2009 and 2013, the downturn in the economy was reflected in a fall from $28 \%$ to $19 \%$ in the proportion of patients who had private status during their delivery episode. The downturn impacted more acutely on delivery discharges than non-delivery inpatient discharges where the fall in the proportion of private discharges over the period was just 3.3 percentage points. The difference here likely reflects the fact that the majority of PHI policies do not cover the entire consultant fee for maternity cases and during times of austerity even those who continued to pay their PHI 

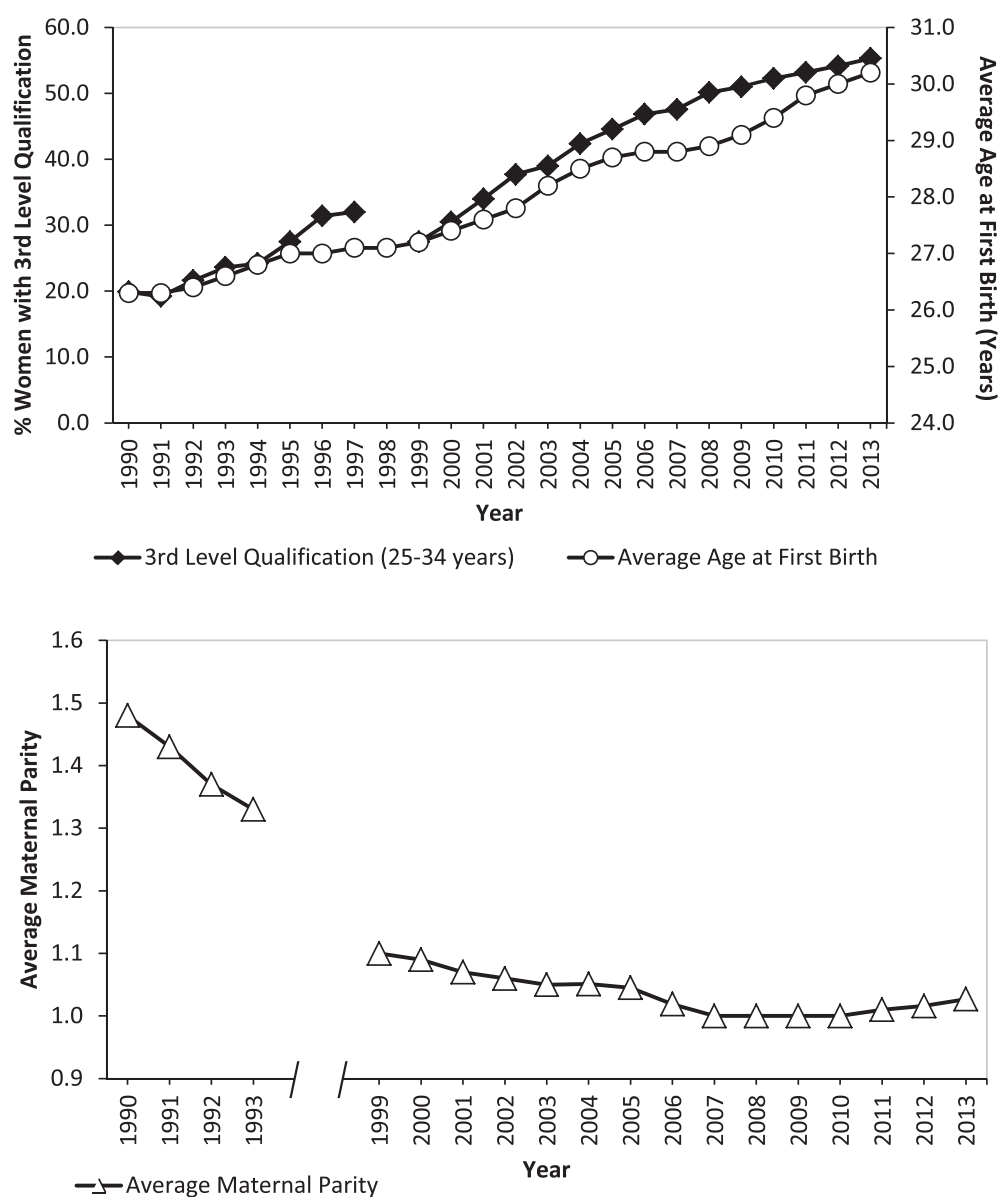

Fig. 2 Average Age at First Birth, Percentage of Women with a 3rd Level Qualification, and Average Maternal Parity in Ireland. Note: No published NPRS data are available for 1994-1998 inclusive. Sources: Education: CSO Women and Men in Ireland, Table 5.42004 and Table 4.4 2013. Consistent series not available prior to 1999. Age: CSO Statbank, Vital Statistics VSA17. Parity: NPRS Annual Reports, 1990-2013

premium could find it difficult to pay the additional consultant fee for private maternity care which can be over $€ 3,000$.

Over the same 5-year period, the ELCS rates for public and private patients have continued to increase $(+40.9 \Delta \%,+38 \Delta \%$ respectively). The latest available national data for 2013 reports an ELCS rate of $13.1 \%$ for public patients and $24.4 \%$ for private patients. This marks a continued increase in the differential between the two groups [44-47]. Provisional work carried out on 2013 data with a more limited set of variables (excluding social class, nationality, birthweight, and previous miscarriage) shows little change in the proportion of the difference for ELCS explained (53.3 \%) and an increase of 9 percentage points in the proportion explained for EMCS. Sensitivity analyses using this limited set of variables for 2009 found no difference to the proportion of the difference explained $(53.1 \%$ in the elective model and $78.7 \%$ in the emergency model) giving us confidence that the more complete analyses using data from 2009 are robust.
Secondly, in June 2010, the Health Service Executive which funds maternity hospitals introduced Clinical Care Programmes for all specialities, including obstetrics and gynaecology. The purpose of the Programmes was to improve clinical input into the health services based on the key metrics of quality, financial value, access and compliance. As part of the Programme, national clinical guidelines have been rolled out including ones on obesity and pregnancy, delivery after caesarean section, and management of pre-eclampsia and eclampsia [48-50]. In addition, each maternity unit has received their own perinatal outcomes (from the NPRS) and performance tables have been developed for CS rates [51]. It is also planned to develop hospital standardised CS rates to allow maternity units to benchmark themselves nationally. It remains to be seen, however, if these quality improvement innovations lead to the standardisation of care and to the reduction in variations in CS rates across models of care.

It is difficult to predict how these developments have impacted on the model of care differential in the ELCS 
and EMCS rate. It is hoped that future work as part of the same project using more recent data, will go some way to explaining this.

\section{Conclusions}

Previous literature has described the increasing prevalence of CS and the role of the private model of care in this. The purpose of this study was to quantify the differential in ELCS and EMCS rates across models of care for singleton nulliparous women and to shed light upon why these differences exist. This is interesting because Ireland is unusual in that private and public patients are delivered in the same delivery suites with the same midwifery staff working with common clinical practices and guidelines. Using national level data from all public hospitals in Ireland, covering almost $97 \%$ of singleton nulliparous births, we find that the majority of the difference in EMCS (80 \%) rates across models of care can be explained by differing characteristics between the two groups of women particularly age. However, the explained component of the difference for ELCS (53\%) is smaller; an excess private effect remains unexplained after accounting for maternal, clinical, and hospital characteristics. Additional, possibly qualitative, studies are recommended to further investigate the reasons for this large and increasing differential.

\section{Additional file}

Additional file 1: Clinical Codes for Risk Factor Identification. ICD-10-AM/ $\mathrm{ACH}$ codes for identification of clinical risk factors in the data. (DOCX $13 \mathrm{~kb}$ )

\section{Abbreviations}

ACHI, Australian Classification of Health Interventions; ACS, Australian Coding Standards; CS, caesarean section; ELCS, elective caesarean section; EMCS, emergency caesarean section; HIPE, hospital in-patient enquiry scheme; ICD-10-AM, the international statistical classification of diseases and related health problems, tenth revision, Australian modification; NPRS, national perinatal reporting system

\section{Acknowledgements}

The authors would like to gratefully acknowledge the assistance of the staff of the National Perinatal Reporting System and the Hospital In-Patient Enquiry Scheme of the Healthcare Pricing Office for making available the data used in this paper and for assistance with clinical coding.

\section{Funding}

This work is funded by the Health Research Board (grant number HRA_HSR/2012/ 8). The study sponsor, had no role in study design; analysis and interpretation of the data; in the writing of the report; and in the decision to submit the paper for publication. All of the researchers are independent from the funders.

\section{Availability of data and materials}

HIPE and NPRS data are available through a data request process from the Healthcare Pricing Office.

\section{Authors' contributions}

$A B, R L$ and $M T$ conceived of the study. $A B, R L$, and $A N$ designed the analyses. $A B$ conducted and interpreted the analyses and drafted the manuscript. RL, $A N$, and MT contributed to interpretation of analyses and critically revised the content of the manuscript. All authors read and approved the final manuscript.

\section{Competing interests}

The authors declare that they have no competing interests.

Consent for publication Not applicable.

Ethics approval and consent to participate

Ethical approval was not required as all data were extracted from anonymised administrative sources for secondary analysis. They were obtained and used with the permission of the Hospital In-Patient Enquiry Scheme, the National Perinatal Reporting System and the Central Statistics Office, Ireland.

\section{Author details}

${ }^{1}$ Economic and Social Research Institute, Whitaker Square, Sir John Rogerson`s Quay, Dublin 2, Ireland. ${ }^{2}$ Trinity College, Dublin, Ireland. ${ }^{3}$ Department of Sociology, Trinity College, Dublin 2, Ireland. ${ }^{4}$ UCD Centre for Human Reproduction, Coombe Women and Infants University Hospital, Dublin 8, Ireland.

Received: 18 December 2015 Revised: 27 June 2016

Accepted: 29 June 2016

Published online: 08 July 2016

\section{References}

1. O'Dwyer V, Farah N, Fattah C, O'Connor N, Kennelly MM, Turner MJ. The risk of caesarean section in obese women analysed by parity. Eur J Obstet Gyn R B. 2011;158(1):28-32.

2. Brick A, Layte $R$ : Recent trends in the caesarean section rate in Ireland 1999-2006. ESRI Working Paper 309. 2009. Available at https://www.esri.ie/ pubs/WP309.pdf

3. Brick A, Layte R. Exploring Trends in the Rate of Caesarean Section in Ireland 1999-2007. Econ Soc Rev. 2011;42(4):383-406.

4. Bayrampour $\mathrm{H}$, Heaman M. Advanced maternal age and the risk of cesarean birth: A systematic review. Birth-Iss Perinat C. 2010;37(3):219-26.

5. Lipkind HS, Duzyj C, Rosenberg TJ, Funai EF, Chavkin W, Chiasson MA. Disparities in cesarean delivery rates and associated adverse neonatal outcomes in New York City hospitals. Obstet Gynecol. 2009;113(6):1239-47.

6. Roberts $\mathrm{CL}$, Tracy S, Peat $\mathrm{B}$. Rates for obstetric intervention among private and public patients in Australia: population based descriptive study. Brit Med J. 2000;321(7254):137-41.

7. Potter JE, Berquó E, Perpétuo IHO, Leal OF, Hopkins K, Souza MR, de Carvalho Formiga MC. Unwanted caesarean sections among public and private patients in Brazil: prospective study. Brit Med J. 2001;323(7322):1155-8.

8. Murray SF. Relation between private health insurance and high rates of caesarean section in Chile: qualitative and quantitative study. Brit Med J. 2000;321(7275):1501-5.

9. Mossialos E, Allin S, Karras K, Davaki K. An investigation of caesarean sections in three Greek hospitals: The impact of financial incentives and convenience. Eur J Public Health. 2005;15(3):288-95.

10. Bertollini R, DiLallo D, Spadea T, Perucci C. Cesarean section rates in Italy by hospital payment mode: an analysis based on birth certificates. Am J Public Health. 1992;82(2):257-61.

11. Declercq $E$, Young $R$, Cabral $H$, Ecker J. Is a rising cesarean delivery rate inevitable? Trends in industrialized countries, 1987 to 2007. Birth-Iss Perinat C. 2011:38(2):99-104

12. Joseph KS, Young DC, Dodds L, O'Connell CM, Allen VM, Chandra S, Allen AC. Changes in maternal characteristics and obstetric practice and recent increases in primary cesarean delivery. Obstet Gynecol. 2003;102(4):791-800.

13. Hanna-Leena Melender R. Experiences of fears associated with pregnancy and childbirth: a study of 329 pregnant women. Birth-Iss Perinat C. 2002;29(2):101-11.

14. Ecker JL, Chen KT, Cohen AP, Riley LE, Lieberman ES. Increased risk of cesarean delivery with advancing maternal age: Indications and associated factors in nulliparous women. Am J Obstet Gyne. 2001;185(4):883-7.

15. Ehrenberg HM, Durnwald CP, Catalano P, Mercer BM. The influence of obesity and diabetes on the risk of cesarean delivery. Am J Obstet Gyne. 2004;191(3):969-74.

16. Cai WW, Marks JS, Chen CH, Zhuang YX, Morris L, Harris JR. Increased cesarean section rates and emerging patterns of health insurance in Shanghai, China. Am J Public Health. 1998;88(5):777-80. 
17. Shorten A, Shorten B. What happens when a private hospital comes to town? The impact of the 'public' to 'private' hospital shift on regional birthing outcomes. Women Birth. 2007;20(2):49-55.

18. Tussing AD, Wojtowycz MA. The cesarean decision in New York State, 1986: Economic and noneconomic aspects. Med Care. 1992;30(6):529-40.

19. Lutomski JE, Murphy M, Devane D, Meaney S, Greene RA. Private health care coverage and increased risk of obstetric intervention. BMC Pregnancy Childbirth 2014:14(13). doi:10.1186/1471-2393-14-13.

20. Murphy DJ. A retrospective cohort study of mode of delivery among public and private patients in an integrated maternity hospital setting. Brit Med J Open, 2013, 3(11). doi:10.1136/bmjopen-2013-003865.

21. Sinnott SJ, Brick A, Layte R, Cunningham N, Turner MJ (2016) National Variation in Caesarean Section Rates: A Cross Sectional Study in Ireland. PLoS ONE 11(6). doi:10.1371/journal.pone.0156172.

22. Grant D. Physician financial incentives and cesarean delivery: new conclusions from the healthcare cost and utilization project. J Health Econ. 2009;28(1):244-50.

23. Gruber J, Owings M. Physician financial incentives and cesarean section delivery. RAND J Econ. 1996;27(1):99-123.

24. National Centre for Classification in Health. Volume 5: Australian Coding Standards for ICD-10-AM. In: The International Statistical Classification of Diseases and Related Health Problems, Tenth Revision, Australian Modification (ICD-10-AM). Sydney: National Centre for Classification in Health; 2004.

25. Euro-Peristat: European Perinatal Health Report: Health and Care of Pregnant Women and Babies in Europe in 2010. 2013. Available at http:// www.europeristat.com/images/doc/EPHR2010_w_disclaimer.pdf

26. Fairlie R. An extension of the Blinder-Oaxaca decomposition technique to logit and probit models. J Econ Soc Meas. 2005;30(4):305-16.

27. Blinder A. Wage discrimination: reduced form and structural estimates. J Hum Resour. 1973;8(4):436-55.

28. Oaxaca R. Male-female wage differentials in urban labor markets. Int Econ Rev. 1973;14(3):693-709.

29. Lhila A, Long S. What is driving the black-white difference in low birthweight in the US? Health Econ. 2012;21(3):301-15.

30. Brick A, Nolan A. Maternal country of birth differences in breastfeeding at hospital discharge in Ireland. Econ Soc Rev. 2014;45(4):455-84.

31. Bhalotra S, Valente C, van Soest A. The puzzle of Muslim advantage in child survival in India. J Health Econ. 2010;29(2):191-204.

32. Panis CW, Lillard LA. Child mortality in Malaysia: explaining ethnic differences and the recent decline. Pop Stud. 1995:49(3):463-79.

33. Wehby G, Murray J, McCarthy A, Castilla E. Racial gaps in child health insurance coverage in four south american countries: The role of wealth, human capital, and other household characteristics. Health Serv Res. 2011; 46(6):2119-38

34. Geruso M. Black-white disparities in life expectancy: How much can the standard SES variables explain? Demography. 2012:49(2):553-74.

35. Costa Font J, Fabbri D, Gil J. Decomposing cross-country differences in levels of obesity and overweight: does the social environment matter? In: LSE Health Working Paper No 12/2008. London: LSE Health; 2008.

36. Fairlie RW. The absence of the African-American owned business: An analysis of the dynamics of self-employment. J Labor Econ. 1999;17(1):80-108.

37. Jann B. Fairlie: Stata module to generate nonlinear decomposition of binary outcome differentials. Boston College Department of Economics: Statistical Software Components; 2006.

38. Fairlie R: Addressing path dependence and incorporating sample weights in the nonlinear blinder-oaxaca decomposition technique for logit, probit and other nonlinear models. University of California, Santa Cruz Working Paper 2015 (June 2015) http://people.ucsc.edu/ rfairlie/decomposition/ decomprevisted $v 9$.docx

39. Einarsdóttir K, Haggar F, Pereira G, Leonard H, de Klerk N, Stanley FJ, Stock S: Role of public and private funding in the rising caesarean section rate: a cohort study. Brit Med J Open 2013:3(5). doi:10.1136/bmjopen-2013-002789.

40. Janssens S, Wallace KL, Chang AMZ. Prepartum and intrapartum caesarean section rates at Mater Mothers' Hospital Brisbane 1997-2005. Aust N Z J Obstet Gynaecol. 2008;48(6):564-9.

41. Tollanes MC, Thompson JM, Daltveit AK, Irgens LM. Cesarean section and maternal education; secular trends in Norway, 1967-2004. Acta Obstet Gyn Scan. 2007;86(7):840-8

42. Bailit JL, Love TE, Mercer B. Rising cesarean rates: Are patients sicker? Am J Obstet Gyne. 2004;191(3):800-3.
43. Barber EL, Lundsberg LS, Belanger K, Pettker CM, Funai EF, Illuzzi JL. Indications contributing to the increasing cesarean delivery rate. Obstet Gynecol. 2011;118(1):29-38.

44. Healthcare Pricing Office. Activity in Acute Public Hospitals in Ireland - 2013 Annual Report. Dublin: Healthcare Pricing Office; 2014.

45. HRID ESRI. Activity in Acute Public Hospitals in Ireland - 2010 Annual Report. Dublin: Economic and Social Research Institute; 2011.

46. HRID ESRI. Activity in Acute Public Hospitals in Ireland - 2011 Annual Report. Dublin: Economic and Social Research Institute; 2012.

47. HRID ESRI. Activity in Acute Public Hospitals in Ireland - 2012 Annual Report. Dublin: Economic and Social Research Institute; 2013.

48. Health Service Executive, Institute of Obstetricians and Gynaecologists. Delivery after previous caesarean section - Clinical practice guideline. Dublin: Health Service Executive; 2013.

49. Health Service Executive, Institute of Obstetricians and Gynaecologists. obesity and pregnancy - Clinical practice guideline. Dublin: Health Service Executive; 2013.

50. Health Service Executive, Institute of Obstetricians and Gynaecologists. The diagnosis and management of pre-eclampsia and eclampsia - Clinical practice guideline. Dublin: Health Service Executive; 2013.

51. Turner MJ. The use of quality control performance charts to analyze cesarean delivery rates nationally. Int J Gynecol Obstet. 2011:113(3):175-7.

\section{Submit your next manuscript to BioMed Central and we will help you at every step:}

- We accept pre-submission inquiries

- Our selector tool helps you to find the most relevant journal

- We provide round the clock customer support

- Convenient online submission

- Thorough peer review

- Inclusion in PubMed and all major indexing services

- Maximum visibility for your research

Submit your manuscript at www.biomedcentral.com/submit
) Biomed Central 